Received: 1 May 2018

Accepted: 19 December 2018

Published online: 20 June 2019

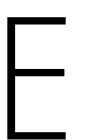

C N

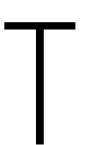

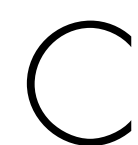

REP

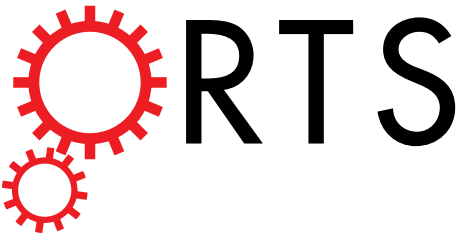

\title{
OPEN
}

\section{Body Fat Percentage in Relation to Lung Function in Individuals with Normal Weight Obesity}

Yuan-Yuei Chen ${ }^{1,2,3}$,Tung-Wei Kao ${ }^{2,3,4,5}$, Wen-Hui Fang 2,3 , Chung-Ching Wang ${ }^{2,3}$,
Yaw-Wen Chang $\mathbb{D}^{2,3,4}$, Hui-Fang Yang ${ }^{2,3,4}$, Chen-Jung Wu $u^{2,3,4,6}$, Yu-Shan Sun ${ }^{2,3,4}$ \&
Wei-Liang Chen $\mathbb{D}^{2,3,4}$

Accumulating evidence indicates the association between obesity and lung function. However, no previous study has examined whether obesity affects lung function in normal weight participants with high body fat. We hypothesized that subjects with normal weight obesity (NWO) were inversely associated with lung function in Taiwan. The study sample was composed of participants who attended health examinations at the Tri-Service General Hospital from 2010 to 2016. A total of 7801 eligible participants who were classified as NWO were divided into quartiles by percentage body fat (PBF), which was measured by bioelectrical impedance analysis (BIA). A multivariable linear regression was performed to assess the association between PBF quartiles and pulmonary function. The relationship between PBF and the presence of obstructive and restrictive lung diseases was analyzed by a logistic regression. PBF quartiles were closely associated with reduced forced expiratory volume in one second (FEV1) and forced vital capacity (FVC) in all adjusted models. This relationship remained significant in the male population, and a dose-dependent effect was observed. Increased PBF was associated with increased risks for the presence of restrictive lung diseases. These results presented a novel finding that body fat exhibited an inverse association with pulmonary function in NWO subjects. More comprehensive management of subjects with normal weight but high body fat, which might contribute to metabolic dysfunction and impaired pulmonary function, is needed.

The prevalence of obesity is progressively increasing worldwide and is becoming a major public health problem in Taiwan $^{1,2}$. Obesity is associated with high risks of insulin resistance ${ }^{3}$, metabolic syndrome ${ }^{4}$, and cardiometabolic diseases ${ }^{5}$, The adverse effect of overweight and obesity on reduced lung function have been observed in numerous studies ${ }^{6,7}$. Body mass index (BMI) was inversely associated with lung function and lung volume ${ }^{8-10}$. In a longitudinal analysis, increased BMI in people who had a BMI $\geq 26.4 \mathrm{~kg} / \mathrm{m}^{2}$ was associated with impaired lung function in young healthy adults ${ }^{11}$. Overweight is potentially associated with decreased forced expiratory volume in one second (FEV1) $)^{12-14}$. An inverse relationship between obesity and forced vital capacity (FVC) was also reported in previous studies ${ }^{15,16}$. The common definition for adiposity or obesity relies on BMI, which is simple and reasonable $^{17}$. However, increased body fat and elevated lean mass are rarely distinguished, particularly in subjects with $\mathrm{BMI}>30 \mathrm{~kg} / \mathrm{m}^{218,19}$.

Normal weight obesity (NWO), an emerging viewpoint initially described by De Lorenzo et al., represents the association between normal weight and high body fat with metabolic dysfunction ${ }^{20}$. Previous evidence

${ }^{1}$ Department of Internal Medicine, Tri-Service General Hospital Songshan Branch; and School of Medicine, National Defense Medical Center, Taipei, Taiwan, Republic of China. ${ }^{2}$ Division of Family Medicine, Department of Family and Community Medicine, Tri-Service General Hospital; and School of Medicine, National Defense Medical Center, Taipei, Taiwan, Republic of China. ${ }^{3}$ Health Management Center, Department of Family and Community Medicine, Tri-Service General Hospital; and School of Medicine, National Defense Medical Center, Taipei, Taiwan, Republic of China. ${ }^{4}$ Division of Geriatric Medicine, Department of Family and Community Medicine, Tri-Service General Hospital; and School of Medicine, National Defense Medical Center, Taipei, Taiwan, Republic of China. ${ }^{5}$ Graduate Institute of Clinical Medical, College of Medicine, National Taiwan University, Taipei, Taiwan, Republic of China. ${ }^{6}$ Division of Family Medicine, Department of Community Medicine, Taoyuan Armed Forces General Hospital, Taoyuan, Taiwan, Republic of China. Correspondence and requests for materials should be addressed to W.-L.C. (email: weiliang0508@ gmail.com) 


\begin{tabular}{|c|c|c|c|c|c|}
\hline Variables & $\begin{array}{l}\text { Q1 } \\
\text { PBF }<21 \\
(N=1952)\end{array}$ & \begin{tabular}{|l} 
Q2 \\
$21<\mathrm{PBF}<26$ \\
$(\mathrm{~N}=1967)$
\end{tabular} & \begin{tabular}{|l}
$\mathrm{Q} 3$ \\
$26<\mathrm{PBF}<31$ \\
$(\mathrm{~N}=1950)$
\end{tabular} & $\begin{array}{l}\text { Q4 } \\
\text { PBF > 31 } \\
(\mathrm{N}=1932)\end{array}$ & P-value \\
\hline \multicolumn{6}{|c|}{ Continuous Variables, mean (SD) } \\
\hline FEV1 (L) & $3.51(0.75)$ & $2.97(0.71)$ & $2.57(0.58)$ & $2.32(0.54)$ & $<0.001$ \\
\hline FVC (L) & $4.08(0.84)$ & $3.47(0.81)$ & $2.99(0.67)$ & $2.70(0.61)$ & $<0.001$ \\
\hline FEV1/FVC & $0.99(0.11)$ & $0.99(0.12)$ & $0.99(0.11)$ & $1.00(0.12)$ & $<0.001$ \\
\hline Age (years) & $42.31(12.74)$ & $46.20(12.40)$ & 46.07 (11.99) & $49.21(13.20)$ & $<0.001$ \\
\hline Height $(\mathrm{cm})$ & $171.57(7.28)$ & $166.36(7.76)$ & $160.91(6.85)$ & $157.99(6.20)$ & $<0.001$ \\
\hline $\mathrm{WC}(\mathrm{cm})$ & $77.89(6.03)$ & $77.90(7.44)$ & $75.93(6.87)$ & $77.71(6.42)$ & $<0.001$ \\
\hline Lean mass (kg) & $29.59(3.91)$ & $25.53(4.99)$ & $21.52(3.39)$ & $19.56(2.96)$ & $<0.001$ \\
\hline $\mathrm{TG}(\mathrm{mg} / \mathrm{dL})$ & $99.70(57.31)$ & $108.42(66.91)$ & $100.50(75.27)$ & $107.33(85.58)$ & $<0.001$ \\
\hline $\mathrm{HDL}-\mathrm{C}(\mathrm{mg} / \mathrm{dL})$ & $54.62(13.52)$ & $54.94(14.37)$ & $59.86(15.09)$ & $59.20(14.02)$ & $<0.001$ \\
\hline FPG (mg/dL) & $89.55(14.82)$ & $92.20(20.87)$ & $90.62(17.99)$ & $92.70(18.14)$ & $<0.001$ \\
\hline $\mathrm{UA}(\mathrm{mg} / \mathrm{dL})$ & $5.91(1.22)$ & $5.49(1.44)$ & $4.80(1.19)$ & $4.82(1.06)$ & $<0.001$ \\
\hline TSH (IU/mL) & $2.15(1.45)$ & $2.26(1.48)$ & $2.28(1.51)$ & $2.56(2.03)$ & $<0.001$ \\
\hline \multicolumn{6}{|c|}{ Category Variables, (\%) } \\
\hline Gender (male) & $1518(91.7)$ & $1020(59.8)$ & $292(17.1)$ & $40(2.3)$ & $<0.001$ \\
\hline Smoking & $768(39.4)$ & $603(30.7)$ & $313(16.1)$ & $155(8.1)$ & $<0.001$ \\
\hline Drinking & $1027(59.5)$ & $860(49.3)$ & $607(35.0)$ & $454(25.8)$ & $<0.001$ \\
\hline
\end{tabular}

Table 1. Characteristics of study sample in normal weight obesity. FEV1, forced expiratory volume in one second; FVC, forced vital capacity; WC, waist circumference; TG, triglyceride; HDL-C, high-density lipoprotein cholesterol; FPG, fasting plasma glucose; UA, uric acid; TSH, thyroid stimulating hormone.

indicates that excess adiposity in NWO individuals might be associated with cardiometabolic disorders, metabolic syndrome, and cardiovascular risk factors ${ }^{21}$. However, no previous study has examined whether obesity influences lung function in an NWO population. The objective of the present study was to investigate the association between body fat percentage (PBF) with lung function measured by pulmonary function tests (PFT) in a cross-sectional study composed of participants who attended health examinations at the Tri-Service General Hospital (TSGH) in Taiwan.

\section{Results}

Characteristics in participants with a normal BMI based on PBF quartiles. The characteristics of 7801 eligible subjects divided by PBF quartiles are provided in Table 1. The mean age was Q1: $42.31 \pm 12.74$ years, Q2: $46.20 \pm 12.40$, Q3: $46.07 \pm 11.99$ and Q4: $49.21 \pm 13.20$, respectively. Participants in higher PBF quartiles exhibited reduced FEV1 and FVC. Demographic factors such as age, height, waist circumference and biochemical data and medical history exhibited significant differences across these PBF quartiles

Pulmonary function in participants with normal BMI based on PBF quartiles. In continuous models (Table 2), PBF quartiles were closely associated with reduced FVC and FEV1 in all adjusted models: FEV1: Q2 $=-0.115(95 \% \mathrm{CI}=-0.156,-0.074), \mathrm{Q} 3=-0.159(95 \% \mathrm{CI}=-0.209,-0.110), \mathrm{Q} 4=-0.164(95 \%$ $\mathrm{CI}=-0.221,-0.107)$; FVC: $\mathrm{Q} 2=-0.118(95 \% \mathrm{CI}=-0.166,-0.070), \mathrm{Q} 3=-0.179(95 \% \mathrm{CI}=-0.237,-0.121)$, $\mathrm{Q} 4=-0.189(95 \% \mathrm{CI}=-0.256,-0.123)$. However, no significant association was observed for FEV1/FVC.

The association between PBF and pulmonary function in based on gender differences is presented in Table 3. The relationship remained significant in the male population and a dose-dependent effect was observed. Specially, higher PBF quartiles exhibited lower pulmonary function in the fully-adjusted model: FEV1: Q2 $=-0.101(95 \%$ $\mathrm{CI}=-0.156,-0.047), \mathrm{Q} 3=-0.169(95 \% \mathrm{CI}=-0.253,-0.085), \mathrm{Q} 4=-0.306(95 \% \mathrm{CI}=-0.510,-0.102)$; FVC: Q2 $=-0.088(95 \% \mathrm{CI}=-0.151,-0.025), \mathrm{Q} 3=-0.177(95 \% \mathrm{CI}=-0.273,-0.081), \mathrm{Q} 4=-0.312(95 \%$ $\mathrm{CI}=-0.547,-0.078)$. By contrast, only the highest PBF quartile exhibited significant associations with reduced $\mathrm{FVC}$ in the female population after fully adjusting for covariables $(\boldsymbol{\beta}=-0.124,95 \% \mathrm{CI}=-0.230,-0.019)$.

Association between PBF and the presence of obstructive and restrictive lung diseases in the NWO population. In Table 4, PBF was significantly associated with the increased risk of restrictive lung diseases in the NWO population with odd ratios (ORs) of 1.029 (95\% CI =1.007-1.050) in the fully-adjusted model. Taking gender into account (Table 5), PBF was associated with increased risk of both obstructive and restrictive lung disease in males after fully adjusting covariables with ORs of $1.112(95 \% \mathrm{CI}=1.001-1.236)$ and $1.042(95 \%$ $\mathrm{CI}=1.009-1.076$ ), respectively. However, no significant difference was found in female participants.

\section{Discussion}

In our cross-sectional study, increased body fat exhibited a detrimental correlation with pulmonary function in an NWO population. Higher PBF quartiles were significantly associated with lower FEV1 and FVC, especially in males. Increased PBF was associated with increased risks for the presence of restrictive lung disease. To the best of our knowledge, our study was the first to examine the relationship between PBF and pulmonary function in NWO participants. 


\begin{tabular}{|c|c|c|c|c|c|c|c|}
\hline Variable & Quartile & Model $^{\mathrm{a}} 1 \beta^{\mathrm{b}}(95 \% \mathrm{CI})$ & $P$ Value & Model $^{\mathrm{a}} 2 \beta^{\mathrm{b}}(95 \% \mathrm{CI})$ & $P$ Value & Model $^{\mathrm{a}} 3 \beta^{\mathrm{b}}(95 \% \mathrm{CI})$ & $P$ Value \\
\hline & \multicolumn{7}{|l|}{ PBF } \\
\hline \multirow{3}{*}{ FEV1 } & Q2 v.s. Q1 & $-0.142(-0.182,-0.102)$ & $<0.001$ & $-0.129(-0.170,-0.089)$ & $<0.001$ & $-0.115(-0.156,-0.074)$ & $<0.001$ \\
\hline & Q3 v.s. Q1 & $-0.191(-0.238,-0.143)$ & $<0.001$ & $-0.181(-0.229,-0.134)$ & $<0.001$ & $-0.159(-0.209,-0.110)$ & $<0.001$ \\
\hline & Q4 v.s. Q1 & $-0.201(-0.253,-0.148)$ & $<0.001$ & $-0.201(-0.253,-0.148)$ & $<0.001$ & $-0.164(-0.221,-0.107)$ & $<0.001$ \\
\hline \multirow{3}{*}{ FVC } & Q2 v.s. Q1 & $-0.149(-0.195,-0.103)$ & $<0.001$ & $-0.139(-0.185,-0.092)$ & $<0.001$ & $-0.118(-0.166,-0.070)$ & $<0.001$ \\
\hline & Q3 v.s. Q1 & $-0.219(-0.275,-0.164)$ & $<0.001$ & $-0.212(-0.267,-0.156)$ & $<0.001$ & $-0.179(-0.237,-0.121)$ & $<0.001$ \\
\hline & Q4 v.s. Q1 & $-0.243(-0.304,-0.182)$ & $<0.001$ & $-0.243(-0.304,-0.182)$ & $<0.001$ & $-0.189(-0.256,-0.123)$ & $<0.001$ \\
\hline \multirow{3}{*}{ FEV1/FVC } & Q2 v.s. Q1 & $-0.003(-0.010,0.004)$ & 0.380 & $-0.003(-0.010,0.004)$ & 0.379 & $-0.005(-0.012,0.002)$ & 0.180 \\
\hline & Q3 v.s. Q1 & $-0.001(-0.009,0.007)$ & 0.762 & $-0.001(-0.009,0.007)$ & 0.758 & $-0.004(-0.013,0.004)$ & 0.328 \\
\hline & Q4 v.s. Q1 & $0.002(-0.007,0.011)$ & 0.732 & $0.002(-0.007,0.011)$ & 0.732 & $-0.003(-0.013,0.007)$ & 0.547 \\
\hline
\end{tabular}

Table 2. Association between PBF and lean mass in quartile and pulmonary function in NWO population. ${ }^{a}$ Adjusted covariates: Model $1=$ age, gender, height. Model $2=$ Model $1+$ age*height. Model $3=$ Model $2+\mathrm{WC}, \mathrm{TG}, \mathrm{HDL}-\mathrm{C}, \mathrm{FPG}, \mathrm{UA}, \mathrm{TSH}+$ history of smoking. ${ }^{\mathrm{b}} \beta$ coefficients is interpreted as change of PBF in different pulmonary function index.

Accumulating evidence indicates that the association between obesity and both lung volume and lung function. Melo et al. demonstrated that reduced lung volume and capacity were present in obese subjects compared with healthy subjects ${ }^{6}$. BMI contributed to reduction in all lung volumes, especially the functional residual capacity $^{8}$. In a study involving three large epidemiological datasets, severe obesity was a risk factor for recent asthma, and both FEV1 and FVC were significantly reduced with increasing BMI ${ }^{22}$. However, no previous study reported the interaction between lung function and individuals with NWO. The concept of NWO was defined as individuals with normal body weight and high $\mathrm{PBF}^{23}$. Emerging evidence indicates that the NWO population was significantly associated with increased risk of developing metabolic syndrome, cardiometabolic diseases and high mortality ${ }^{21}$. In a cross-sectional study of a representative sample of US adults, Chen et al. reported that increased number of metabolic syndrome components and individual components of metabolic syndrome exhibited a relationship with impaired FEV1 and $\mathrm{FVC}^{24}$. This finding strongly supported the viewpoint of our study and implied a plausible explanation for reduced lung function in the NWO population.

Lung function impairment is associated with increased PBF in obese individuals ${ }^{25}$. Similar results were presented in a study of elderly subjects, demonstrating that increased PBF had a negative impact on lung function in both genders ${ }^{26}$. These findings were consistent with our study findings suggesting that increased PBF was detrimental to lung function and related to several pulmonary diseases. Several potential mechanisms of the interaction between PBF and lung function are noted. Adiposity accumulation in the thoracic and abdominal regions might directly affect the downward movement of the diaphragm and chest wall properties ${ }^{7}$. Reduced lung volume resulted from the android pattern of body fat deposition by generating increased resistance to diaphragmatic contraction and impairing respiratory mechanics ${ }^{27}$. In addition to the mechanical pathway, the metabolic dysfunction caused by adipose tissue might contribute to the inverse effect. As the core pathophysiology of metabolic syndrome ${ }^{3}$, abdominal obesity was identified as the main factor by which increased C-reactive protein (CRP) concentrations caused by visceral fat accumulation ${ }^{28}$. Systemic inflammation might lead to reduced lung function given that CRP and interleukin-6 (IL-6) expressed in inflammatory lung epithelial cells ${ }^{29}$. Chronic obstructive pulmonary disease, a major cause of reduced FEV1, resulted from lung inflammation related to high serum levels of $\mathrm{CRP}^{30,31}$. Another plausible mechanism was that increased levels of cytokines, particularly IL-6, caused inflammatory cell infiltration into the pulmonary capillary endothelium and altered endothelial function ${ }^{32}$.

Although our study had the strengths of a large population-based survey and a novel finding regarding the impact of body composition on lung function in NWO individuals, the present analysis still has some potential limitations. First, causation between PBF and lung function was not determined due to the cross-sectional design. Long-term observations should be performed in future studies. Second, the study sample was obtained from a single medical center composed of Asian adult. Thus, the limited ethnic distribution of participants might not represent the effect of PBF on lung function in terms of racial differences. Finally, PBF was measured by BIA in the present study. Dual-energy X-ray absorptiometry (DEXA), which is the golden standard for body composition measurement internationally might be more accurate than BIA.

\section{Conclusion}

The present study highlighted a novel finding that PBF was detrimentally correlated with lung function in an NWO population. The concept of NWO reflected the effect of metabolic dysregulation and systemic inflammation caused by increased PBF that might not detected by BMI. The relationship between lung function and the general definition of obesity based on weight and height has been challenged given that individuals with normal weight and high PBF exhibited the same results, and this effect might be more closely associated with the reduction of lung function. A better understanding and recognition of the complex interaction between body compositions and the pathophysiology of lung function should be addressed in the future studies. 


\begin{tabular}{|c|c|c|c|c|c|c|c|c|}
\hline Gender & Variable & Quartile & Model $^{\mathrm{a}} 1 \beta^{\mathrm{b}}(95 \% \mathrm{CI})$ & $P$ Value & Model $^{\mathrm{a}} 2 \beta^{\mathrm{b}}(95 \% \mathrm{CI})$ & $P$ Value & Model $^{\mathrm{a}} 3 \beta^{\mathrm{b}}(95 \% \mathrm{CI})$ & $P$ Value \\
\hline & & PBF & & & & & & \\
\hline \multirow{9}{*}{ Male } & \multirow{3}{*}{ FEV1 } & Q2 v.s. Q1 & $-0.118(-0.169,-0.067)$ & $<0.001$ & $-0.119(-0.170,-0.067)$ & $<0.001$ & $-0.101(-0.156,-0.047)$ & $<0.001$ \\
\hline & & \begin{tabular}{|l|} 
Q3 v.s. Q1 \\
\end{tabular} & $-0.197(-0.275,-0.118)$ & $<0.001$ & $-0.196(-0.274,-0.118)$ & $<0.001$ & $-0.169(-0.253,-0.085)$ & $<0.001$ \\
\hline & & Q4 v.s. Q1 & $-0.347(-0.547,-0.146)$ & $<0.001$ & $-0.344(-0.544,-0.143)$ & $<0.001$ & $-0.306(-0.510,-0.102)$ & 0.003 \\
\hline & \multirow{3}{*}{ FVC } & Q2 v.s. Q1 & $-0.118(-0.177,-0.059)$ & $<0.001$ & $-0.118(-0.178,-0.058)$ & $<0.001$ & $-0.088(-0.151,-0.025)$ & 0.006 \\
\hline & & Q3 v.s. Q1 & $-0.229(-0.319,-0.138)$ & $<0.001$ & $-0.228(-0.318,-0.138)$ & $<0.001$ & $-0.177(-0.273,-0.081)$ & $<0.001$ \\
\hline & & Q4 v.s. Q1 & $-0.381(-0.611,-0.150)$ & $<0.001$ & $-0.378(-0.609,-0.147)$ & $<0.001$ & $-0.310(-0.545,-0.076)$ & 0.010 \\
\hline & \multirow{3}{*}{ FEV1/FVC } & Q2 v.s. Q1 & $-0.004(-0.012,0.004)$ & 0.303 & $-0.005(-0.012,0.003)$ & 0.257 & $-0.008(-0.017,0.000)$ & 0.060 \\
\hline & & \begin{tabular}{|l|} 
Q3 v.s. Q1 \\
\end{tabular} & $-0.003(-0.015,0.009)$ & 0.651 & $-0.002(-0.015,0.010)$ & 0.685 & $-0.009(-0.022,0.004)$ & 0.182 \\
\hline & & Q4 v.s. Q1 & $-0.020(-0.051,0.011)$ & 0.211 & $-0.018(-0.049,0.013)$ & 0.249 & $-0.027(-0.059,0.004)$ & 0.087 \\
\hline \multirow{9}{*}{ Female } & \multirow{3}{*}{ FEV1 } & Q2 v.s. Q1 & $-0.014(-0.105,0.077)$ & 0.761 & $-0.015(-0.105,0.076)$ & 0.752 & $-0.011(-0.101,0.080)$ & 0.817 \\
\hline & & Q3 v.s. Q1 & $-0.061(-0.148,0.026)$ & 0.169 & $-0.062(-0.149,0.025)$ & 0.162 & $-0.047(-0.134,0.040)$ & 0.289 \\
\hline & & Q4 v.s. Q1 & $-0.097(-0.184,-0.011)$ & 0.027 & $-0.097(-0.184,-0.011)$ & 0.028 & $-0.063(-0.152,0.025)$ & 0.161 \\
\hline & \multirow{3}{*}{ FVC } & Q2 v.s. Q1 & $-0.054(-0.162,0.054)$ & 0.328 & $-0.055(-0.163,0.053)$ & 0.316 & $-0.049(-0.157,0.059)$ & 0.372 \\
\hline & & Q3 v.s. Q1 & $-0.114(-0.217,-0.011)$ & 0.031 & $-0.116(-0.219,-0.013)$ & 0.028 & $-0.097(-0.201,0.006)$ & 0.066 \\
\hline & & Q4 v.s. Q1 & $-0.165(-0.268,-0.062)$ & 0.002 & $-0.165(-0.268,-0.062)$ & 0.002 & $-0.124(-0230,-0.019)$ & 0.021 \\
\hline & \multirow{3}{*}{ FEV1/FVC } & Q2 v.s. Q1 & $0.010(-0.008,0.028)$ & 0.263 & $0.010(-0.007,0.028)$ & 0.258 & $0.010(-0.008,0.027)$ & 0.290 \\
\hline & & Q3 v.s. Q1 & $0.011(-0.006,0.028)$ & 0.192 & $0.011(-0.005,0.028)$ & 0.186 & $0.010(-0.007,0.027)$ & 0.234 \\
\hline & & Q4 v.s. Q1 & $0.014(-0.003,0.031)$ & 0.104 & $0.014(-0.003,0.031)$ & 0.104 & $0.012(-0.005,0.030)$ & 0.154 \\
\hline
\end{tabular}

Table 3. Association between PBF in quartile and pulmonary function tests in NWO population in gender

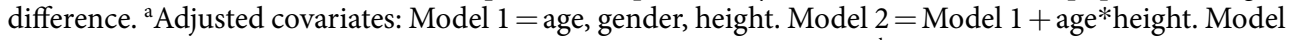
$3=$ Model $2+$ WC, TG, HDL-C, FPG, UA, TSH + history of smoking. ${ }^{\mathrm{b}} \beta$ coefficients is interpreted as change of $\mathrm{PBF}$ in different pulmonary function index.

\section{Methods}

Study design and participants selection. A cohort study involving participants aged 20 years old and older who attended health examinations at the TSGH from 2010 to 2016 was performed. Participants who were underweight $(\mathrm{BMI}<18.5)$, overweight $(24<\mathrm{BMI}<27)$, and obese $(\mathrm{BMI}>27)$ were excluded initially. Those with missing data such as biochemical examinations $(\mathrm{n}=6182)$, body composition measurement $(\mathrm{n}=21424)$, and PFT $(\mathrm{n}=34519)$ were also excluded. The remaining 7801 participants were defined as an NWO population and divided into PBF quartiles: $\mathrm{Q} 1=\mathrm{PBF}<21, \mathrm{Q} 2=21<\mathrm{PBF}<26, \mathrm{Q} 3=26<\mathrm{PBF}<31, \mathrm{Q} 4=\mathrm{PBF}>31$. Study approval was obtained from the Institutional Review Board of TSGH, Taiwan. The TSGH IRB waived the informed consent because these data were analyzed anonymously. All methods were performed in accordance with the relevant guidelines and regulations of TSGH.

Pulmonary function test and quality control. PFT measurements and results interpretation were defined by guidelines published by the European Respiratory and American Thoracic Societies ${ }^{33-35}$. Spirometry is a commonly used method for detecting lung function that represents a measure of volume against time. Subjects were requested to inhale forcefully and then to exhale vigorously for as long and as quickly as possible. Measurement of pulmonary mechanics assesses the ability of the lungs to move huge volumes of air quickly through the airways to identify airway obstruction. The entire study was continuously monitored by well-trained technicians, and a physician regularly supervised them and provided additional instructions. Furthermore, a senior physician reviewed the raw flow volume and volume-time curves and graded the subject's performance. Data that were unacceptable as judged by a senior physician were excluded from the study. In the current study, the characteristics of PFT obtained from the study sample, including FEV1, FVC and the ratio of the two volumes (FEV1/FVC), were assessed in the analysis. The predicted values for FEV1 and FVC were calculated from the following equations obtained in a non-smoking and healthy Taiwanese adult sample ${ }^{36}$.

(1) Predicted FEV1

Men: 95.502-0.280 (Age)

Women: 96.991-0.291(Age)

(2) Predicted FVC

Men: $-2.058+0.0456$ (Height)-0.0246(Age)

Women: $-2.314+0.038$ (Height)-0.0173(Age)

Definition of obstructive and restrictive lung diseases. Obstructive lung disease is a type of respiratory disease characterized by airway obstruction, including asthma, bronchiectasis and chronic obstructive pulmonary disease. Restrictive lung disease is a category of extrapulmonary, pleural, or parenchymal respiratory diseases that restrict lung expansion, leading to decreased lung volume and inadequate ventilation and/or oxygenation. Obstructive lung disease was identified as a FEV1/FVC $<70 \%$ where FEV1 was reduced more than FVC. Restrictive lung disease was defined as a FEV1/FVC $>70 \%$ where FVC was reduced more than FEV $1^{37}$. 


\begin{tabular}{|c|c|c|c|c|c|c|}
\hline Outcomes & Model $^{\mathrm{a}} 1 \mathrm{OR}^{\mathrm{b}}(95 \% \mathrm{CI})$ & $P$ Value & Model $^{\mathrm{a}} 2 \mathrm{OR}^{\mathrm{b}}(95 \% \mathrm{CI})$ & $P$ Value & Model $^{\mathrm{a}} 3 \mathrm{OR}^{\mathrm{b}}(95 \% \mathrm{CI})$ & $P$ Value \\
\hline & \multicolumn{6}{|l|}{ PBF } \\
\hline Obstructive & $1.022(0.957-1.092)$ & 0.518 & $1.022(0.956-1.092)$ & 0.521 & $1.044(0.969-1.125)$ & 0.256 \\
\hline Restrictive & $1.045(1.026-1.065)$ & $<0.001$ & $1.046(1.026-1.065)$ & $<0.001$ & $1.029(1.007-1.050)$ & 0.008 \\
\hline
\end{tabular}

Table 4. Association between PBF and the presence of obstructive and restrictive lung defects in NWO population. ${ }^{a}$ Adjusted covariates: Model $1=$ age, gender, height. Model $2=$ Model $1+$ age $*$ height. Model $3=$ Model 2 + WC, TG, HDL-C, FPG, UA, TSH + history of smoking. ${ }^{\mathrm{b}} \mathrm{OR}$ is interpreted as change of PBF in different pulmonary diseases.

\begin{tabular}{|c|c|c|c|c|c|c|c|}
\hline Gender & Outcomes & Model $^{\mathrm{a}} 1 \mathrm{OR}^{\mathrm{b}}(95 \% \mathrm{CI})$ & $P$ Value & Model $^{\mathrm{a}} 2 \mathrm{OR}^{\mathrm{b}}(95 \% \mathrm{CI})$ & $P$ Value & Model $^{\mathrm{a}} 3 \mathrm{OR}^{\mathrm{b}}(\mathbf{9 5} \% \mathrm{CI})$ & $P$ Value \\
\hline & & \multicolumn{6}{|l|}{ PBF } \\
\hline \multirow{2}{*}{ Male } & Obstructive & $1.046(0.946-1.157)$ & 0.377 & $1.054(0.952-1.167)$ & 0.314 & $1.112(1.001-1.236)$ & 0.049 \\
\hline & Restrictive & $1.058(1.029-1.088)$ & $<0.001$ & $1.059(1.030-1.088)$ & $<0.001$ & $1.042(1.009-1.076)$ & 0.011 \\
\hline \multirow{2}{*}{ Female } & Obstructive & $1.003(0.921-1.091)$ & 0.952 & $1.004(0.922-1.094)$ & 0.925 & $1.001(0.908-1.104)$ & 0.981 \\
\hline & Restrictive & $1.034(1.008-1.061)$ & 0.010 & $1.033(1.007-1.059)$ & 0.014 & $1.016(0.988-1.045)$ & 0.268 \\
\hline
\end{tabular}

Table 5. Association between $\mathrm{PBF}$ and the presence of obstructive and restrictive lung diseases in NWO population in gender difference. ${ }^{a}$ Adjusted covariates: Model $1=$ age, gender, height. Model $2=$ Model

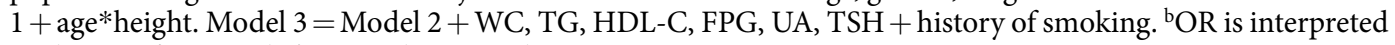
as change of PBF in different pulmonary diseases.

Measurement of body composition. PBF was measured by BIA (InBody720, Biospace, Inc., Cerritos, CA, USA), an useful method for assessing body composition ${ }^{38}$. The BIA procedure was simple and noninvasive, and the results were reproducible and rapidly obtained.

Covariates measurement. Information regarding cigarette smoking was obtained from participants by asking the following question: "How many packs do you smoke per day?". BMI was estimated based on a general formula wherein the weight of the individual in kilograms was divided by the square of the height in meters $\left(\mathrm{kg} / \mathrm{m}^{2}\right)$. Biochemical data were collected by drawing blood samples from subjects after fasting for at least 8 hours and were measured by respective standard procedures.

Statistical analysis. Statistical Package for the Social Sciences, version18.0 (SPSS Inc., Chicago, IL, USA) for Windows was employed for statistical estimations in the study. Student's t test and Pearson's chi-square test were used to examine the differences in terms of demographic information and biochemical data between PBF quartiles. The threshold for statistical significance was defined as a two-sided $p$-value of $\leq 0.05$. Multivariable adjustment for related clinical variables was performed in an extend-model approach. Model 1: age, gender, and height; Model 2: Model l plus age x height; Model 3: Model 2 plus waist circumference (WC), triglyceride (TG), high density lipoprotein cholesterol (HDL-C), fasting plasma glucose (FPG), uric acid (UA), thyroid stimulating hormone (TSH), and history of smoking. Multivariable linear regression was applied to assess the association between $\mathrm{PBF}$ based on quartiles with pulmonary function parameters. Logistic regression was performed to assess the association between $\mathrm{PBF}$ and the presence of obstructive and restrictive lung diseases.

\section{References}

1. Hwang, L. C., Bai, C. H. \& Chen, C. J. Prevalence of obesity and metabolic syndrome in Taiwan. Journal of the Formosan Medical Association = Taiwan yi zhi 105, 626-635, https://doi.org/10.1016/s0929-6646(09)60161-3 (2006).

2. Chu, N. F. Prevalence of obesity in Taiwan. Obes Rev 6, 271-274, https://doi.org/10.1111/j.1467-789X.2005.00175.x (2005).

3. Despres, J. P. \& Lemieux, I. Abdominal obesity and metabolic syndrome. Nature 444, 881-887, https://doi.org/10.1038/nature05488 (2006).

4. Kahn, B. B. \& Flier, J. S. Obesity and insulin resistance. Journal of Clinical Investigation 106, 473-481 (2000).

5. Aronne, L. J. \& Isoldi, K. K. Overweight and obesity: key components of cardiometabolic risk. Clinical cornerstone 8, 29-37 (2007).

6. Melo, L. C., da Silva, M. A. M. \& Calles, A. C. N. Obesity and lung function: a systematic review. Einstein 12, 120-125, https://doi. org/10.1590/s1679-45082014rw2691 (2014).

7. Salome, C. M., King, G. G. \& Berend, N. Physiology of obesity and effects on lung function. Journal of applied physiology (Bethesda, Md.: 1985) 108, 206-211, https://doi.org/10.1152/japplphysiol.00694.2009 (2010)

8. Jones, R. L. \& Nzekwu, M. M. The effects of body mass index on lung volumes. Chest 130, 827-833, https://doi.org/10.1378/ chest.130.3.827 (2006).

9. Costa, D., Barbalho, M. C., Miguel, G. P. S., Forti, E. M. P. \& Azevedo, J. L. M. C. The Impact of Obesity on Pulmonary Function in Adult Women. Clinics (Sao Paulo, Brazil) 63, 719-724, https://doi.org/10.1590/S1807-59322008000600002 (2008).

10. Banerjee, J. et al. Association of Body Mass Index (BMI) with Lung Function Parameters in Non-asthmatics Identified by Spirometric Protocols. Journal of Clinical and Diagnostic Research: JCDR 8, 12-14, https://doi.org/10.7860/JCDR/2014/7306.3993 (2014).

11. Thyagarajan, B. et al. Longitudinal association of body mass index with lung function: The CARDIA Study. Respiratory Research 9 , 31, https://doi.org/10.1186/1465-9921-9-31 (2008).

12. Zerah, F. et al. Effects of obesity on respiratory resistance. Chest 103, 1470-1476 (1993). 
13. Carey, I. M., Cook, D. G. \& Strachan, D. P. The effects of adiposity and weight change on forced expiratory volume decline in a longitudinal study of adults. International journal of obesity and related metabolic disorders: journal of the International Association for the Study of Obesity 23, 979-985 (1999).

14. Steele, R. M., Finucane, F. M., Griffin, S. J., Wareham, N. J. \& Ekelund, U. Obesity is associated with altered lung function independently of physical activity and fitness. Obesity (Silver Spring, Md.) 17, 578-584, https://doi.org/10.1038/oby.2008.584 (2009).

15. Canoy, D. et al. Abdominal Obesity and Respiratory Function in Men and Women in the EPIC-Norfolk Study, United Kingdom. American Journal of Epidemiology 159, 1140-1149, https://doi.org/10.1093/aje/kwh155 (2004).

16. Saliman, J. A. et al. Pulmonary function in the morbidly obese. Surgery for obesity and related diseases: official journal of the American Society for Bariatric Surgery 4, 632-639; discussion 639, https://doi.org/10.1016/j.soard.2008.06.010 (2008).

17. Flegal, K. M., Graubard, B. I., Williamson, D. F. \& Gail, M. H. Cause-specific excess deaths associated with underweight, overweight, and obesity. Jama 298, 2028-2037, https://doi.org/10.1001/jama.298.17.2028 (2007).

18. Romero-Corral, A. et al. Accuracy of body mass index in diagnosing obesity in the adult general population. International journal of obesity (2005) 32, 959-966, https://doi.org/10.1038/ijo.2008.11 (2008).

19. Romero-Corral, A. et al. Association of bodyweight with total mortality and with cardiovascular events in coronary artery disease: a systematic review of cohort studies. Lancet (London, England) 368, 666-678, https://doi.org/10.1016/s0140-6736(06)69251-9 (2006).

20. De Lorenzo, A., Martinoli, R., Vaia, F. \& Di Renzo, L. Normal weight obese (NWO) women: an evaluation of a candidate new syndrome. Nutrition, metabolism, and cardiovascular diseases: NMCD 16, 513-523, https://doi.org/10.1016/j.numecd.2005.10.010 (2006).

21. Romero-Corral, A. et al. Normal weight obesity: a risk factor for cardiometabolic dysregulation and cardiovascular mortality. European heart journal 31, 737-746, https://doi.org/10.1093/eurheartj/ehp487 (2010).

22. Schachter, L., Salome, C., Peat, J. \& Woolcock, A. Obesity is a risk for asthma and wheeze but not airway hyperresponsiveness. Thorax 56, 4-8, https://doi.org/10.1136/thorax.56.1.4 (2001).

23. Oliveros, E., Somers, V. K., Sochor, O., Goel, K. \& Lopez-Jimenez, F. The concept of normal weight obesity. Progress in cardiovascular diseases 56, 426-433, https://doi.org/10.1016/j.pcad.2013.10.003 (2014).

24. Chen, W. L. et al. Relationship between lung function and metabolic syndrome. PloS one 9 , e108989, https://doi.org/10.1371/journal. pone.0108989 (2014)

25. Kamal, R., Kesavachandran, C. N., Bihari, V., Sathian, B. \& Srivastava, A. K. Alterations in Lung Functions Based on BMI and Body Fat \% Among Obese Indian Population at National Capital Region. Nepal Journal of Epidemiology 5, 470-479, https://doi. org/10.3126/nje.v5i2.12829 (2015).

26. Karacan, S., Guzel, N. A., Colakoglu, F. \& Baltaci, G. Relationship between body composition and lung function in elderly men and women. Advances in therapy 25, 168-178, https://doi.org/10.1007/s12325-008-0022-1 (2008).

27. Mafort, T. T., Rufino, R., Costa, C. H. \& Lopes, A. J. Obesity: systemic and pulmonary complications, biochemical abnormalities, and impairment of lung function. Multidisciplinary Respiratory Medicine 11, 28, https://doi.org/10.1186/s40248-016-0066-z (2016).

28. Faber, D. R., van der Graaf, Y., Westerink, J. \& Visseren, F. L. Increased visceral adipose tissue mass is associated with increased C-reactive protein in patients with manifest vascular diseases. Atherosclerosis 212, 274-280, https://doi.org/10.1016/j.atherosclerosis.2010.04.029 (2010).

29. Gould, J. M. \& Weiser, J. N. Expression of C-Reactive Protein in the Human Respiratory Tract. Infection and Immunity 69, 1747-1754, https://doi.org/10.1128/IAI.69.3.1747-1754.2001 (2001).

30. Pauwels, R. A., Buist, A. S., Calverley, P. M., Jenkins, C. R. \& Hurd, S. S. Global strategy for the diagnosis, management, and prevention of chronic obstructive pulmonary disease. NHLBI/WHO Global Initiative for Chronic Obstructive Lung Disease (GOLD) Workshop summary. American journal of respiratory and critical care medicine 163, 1256-1276, https://doi.org/10.1164/ ajrccm.163.5.2101039 (2001).

31. Eid, A. A. et al. Inflammatory response and body composition in chronic obstructive pulmonary disease. American journal of respiratory and critical care medicine 164, 1414-1418, https://doi.org/10.1164/ajrccm.164.8.2008109 (2001).

32. Pittet, J. F., Mackersie, R. C., Martin, T. R. \& Matthay, M. A. Biological markers of acute lung injury: prognostic and pathogenetic significance. American journal of respiratory and critical care medicine 155, 1187-1205, https://doi.org/10.1164/ajrccm.155.4.9105054 (1997).

33. Miller, M. R. et al. General considerations for lung function testing. The European respiratory journal 26, 153-161, https://doi.org/1 0.1183/09031936.05.00034505 (2005).

34. Pellegrino, R. et al. Interpretative strategies for lung function tests. The European respiratory journal 26, 948-968, https://doi.org/10 $.1183 / 09031936.05 .00035205$ (2005).

35. Lung function testing: selection of reference values and interpretative strategies. American Thoracic Society. The American review of respiratory disease 144, 1202-1218, https://doi.org/10.1164/ajrccm/144.5.1202 (1991).

36. Pan, W. H. et al. Reference spirometric values in healthy Chinese neversmokers in two townships of Taiwan. The Chinese journal of physiology 40, 165-174 (1997).

37. Ranu, H., Wilde, M. \& Madden, B. Pulmonary Function Tests. The Ulster Medical Journal 80, 84-90 (2011).

38. Sergi, G., De Rui, M., Stubbs, B., Veronese, N. \& Manzato, E. Measurement of lean body mass using bioelectrical impedance analysis: a consideration of the pros and cons. Aging clinical and experimental research 29, 591-597, https://doi.org/10.1007/s40520-0160622-6 (2017).

\section{Acknowledgements}

This research did not receive any specific grant from any funding agency in the public, commercial or not-forprofit sector.

\section{Author Contributions}

Yuan-Yuei Chen contributed to the design of the study, was responsible for the management and retrieval of data, contributed to initial data analysis and interpretation, drafted the initial manuscript. Yuan-Yuei Chen, TungWei Kao, Wen-Hui Fang, Chung-Ching Wang, Yaw-Wen Chang, Hui-Fang Yang, Chen-Jung Wu, Yu-Shan Sun, Wei-Liang Chen decided upon the data collection methods. Yuan-Yuei Chen and Wei-Liang Chen were also responsible for the data analysis decisions. Wei-Liang Chen conceptualized and designed the study, supervised all aspects of the study, critically reviewed and revised the manuscript, and approved the final manuscript as submitted. All authors meet the ICMJE criteria for authorship.

\section{Additional Information}

Competing Interests: The authors declare no competing interests.

Publisher's note: Springer Nature remains neutral with regard to jurisdictional claims in published maps and institutional affiliations. 
(i) Open Access This article is licensed under a Creative Commons Attribution 4.0 International License, which permits use, sharing, adaptation, distribution and reproduction in any medium or format, as long as you give appropriate credit to the original author(s) and the source, provide a link to the Creative Commons license, and indicate if changes were made. The images or other third party material in this article are included in the article's Creative Commons license, unless indicated otherwise in a credit line to the material. If material is not included in the article's Creative Commons license and your intended use is not permitted by statutory regulation or exceeds the permitted use, you will need to obtain permission directly from the copyright holder. To view a copy of this license, visit http://creativecommons.org/licenses/by/4.0/.

(C) The Author(s) 2019 